\title{
Does obstructive sleep apnea impact the sleep quality, physical capacity, mood and quality of life of elderly lean Men?
}

\begin{abstract}
Objective: The aim of this study was to compare the sleep quality, daytime sleepiness, exercise capacity, mood and quality of life of lean elderly men with Obstructive Sleep Apnea (OSA) vs non-sleep apnea.

Methods: Forty untrained men, between 65 and 75 years old, were allocated into two groups: OSA group (apnea-hypopnea index-AHI $\geq 15 ; \mathrm{n}=21$ ) or control group $(\mathrm{AHI}<15$; $\mathrm{n}=19$ ). All the participants underwent polysomnography and cardiopulmonary exercise testing (CPET). Moreover, all participants completed the Epworth Sleepiness Scale (ESS), Pittsburgh Sleep Quality Index (PSQI), Quality of life (SF-36) and Mood Profile (POMS).

Results: There were no significant differences between the groups in the sleep quality, exercise capacity, mood and daytime sleepiness. A slight effect was observed in the quality of life. The general health perception was enhanced in the control compared with OSA group (control: $87.5 \pm 12.8$ vs. OSA: $74.5 \pm 16.6, \mathrm{p}<0,05$ ); this SF-36 factor was significantly associated with the apnea/hypopnea index $(\mathrm{r}=-0.39 ; \mathrm{p}<0,05)$.

Conclusion: Despite a slight effect on the quality of life, the general results of this study suggest that OSA does not impact significantly sleep quality, physical capacity, mood and quality of life in lean elderly men. We could hypothesize that daytime impairments in OSA patients are mediated by body mass index.
\end{abstract}

Keywords: functional capacity, sleep, polysomnography, quality of life, exercise
Volume I Issue 3 - 2017

\author{
Marcos Gonçalves de Santana, ${ }^{1,2}$ Giselle \\ Soares Passos, ${ }^{1,2}$ Rita Aurélia Boscolo,' Valter \\ Rocha Viana,' Viviane Grassmann,' Sergio \\ Tufik,' Marco Túlio de Mello',3 \\ 'Department of Psychobiology, Universidade Federal de São \\ Paulo, Brazil \\ ${ }^{2}$ Department of Health Applied Science, Universidade Federal \\ de Goias-Regional Jataí, Brazil \\ ${ }^{3}$ Department of Physical Education, Universidade Federal de \\ Minas Gerais, Brazil
}

Correspondence: Giselle Soares Passos, Universidade Federal de Goias, BR 364, n 3800, CEP 7580 I-6I5, Goiás, Brazil,Tel +55643606-8304, Fax +55643606-8304,

Email passos.gs@gmail.com

Received:September 23, 2017 | Published: November 21, 2017
Abbreviations: 1-RM, one maximum repetition; AHI, apnea/ hypopnea index; BMI, body mass index; CPET, cardiopulmonary exercise testing; ESS, epworth sleepiness scale; HR, heart rate; MMSE, mini mental state examination; NREM, non rapid eye movement; OSA, obstructive sleep apnea; PLM, periodic leg movements; POMS, profile of mood states; PSG, polysomnographic; REM, rapid eye movement; $\mathrm{SaO} 2$, arterial oxygen saturation; SE, sleep efficiency; SF36, short form-36; SOL, sleep onset latency; TTS, total sleep time; $\dot{V}$ $\mathrm{O}_{2}$, oxygen uptake; WASO, wake after sleep onset

\section{Introduction}

Obstructive sleep apnea (OSA) is one of the most common sleep disorders around the world. An epidemiologic Brazilian study found that $32.8 \%$ of the people in São Paulo have an OSA clinical diagnosis. ${ }^{1}$ In addition; several studies have found a greater prevalence of OSA in people older than 60 years of age. ${ }^{1,2}$ Some symptoms are directly associated with OSA; such as excessive daytime sleepiness; snoring; fatigue; cognitive impairments and poor quality of sleep. Cardiovascular mortality and hypertension have been correlated with $\mathrm{OSA}^{3}$ and are directly associated with obesity. ${ }^{4}$

Obesity is directly related to a higher prevalence of OSA and is associated with impairments in exercise capacity. ${ }^{5,6}$ A recent study found that lean middle-aged subjects with OSA have no loss of functional capacity or exercise performance. The authors suggested that obesity most likely contributes to the diminished cardiopulmonary capacity in middle-aged subjects with OSA. ${ }^{7}$
Some studies have suggested that OSA in the elderly may be a distinct condition from OSA in the middle-aged. ${ }^{2}$ To our knowledge; there are no studies about OSA-induced impairments in the exercise capacity of lean elderly subjects. Furthermore; many previous studies have demonstrated that OSA in the middle-aged induces excessive daytime sleepiness; mood problems and poor quality of life; ${ }^{8-10}$ but there is no evidence demonstrating a relationship between OSA and body mass in the elderly. Thus; our aim was to compare the sleep quality; daytime sleepiness; exercise capacity; mood and quality of life of lean elderly men with Obstructive Sleep Apnea (OSA) vs nonsleep apnea.

\section{Methods}

All experimental procedures were approved by University Human Research Ethics Committee conformed to the principles outlined in the Declaration of Helsinki. The participants were recruited from the Geriatric Clinic of Federal University of Sao Paulo; Brazil. The initial inclusion criteria were: being a healthy male between 65 and 75 years old; untrained and not a current smoker. The initial exclusions included: severe physical impairments; body mass index $(\mathrm{BMI})>27 \mathrm{~kg} \cdot \mathrm{m}^{-2.11}$ periodic leg movements $>15$ events $/$ hour $^{3}{ }^{3}$ a Mini Mental State Examination score $<26 ;{ }^{12}$ neurological or psychiatric diseases; alcohol abuse and/or psychoactive drugs use and less than 8 years of schooling. An apnea-hypopnea index (AHI) $\geq 15$ was considered diagnostic for OSA. The control group was formed by those with $A H I<15$. These cut-off values were based on previous studies of OSA in elderly patients. ${ }^{2,3}$ All prospective participants were submitted in a clinical evaluation and an electrocardiogram at rest and 
under maximal stress. Participants with any cardiovascular problem could be a risk factor for exercise was not included in the study.

\section{Experimental measures}

Polysomnography: The volunteers underwent polysomnographic (PSG) recording at the Sleep Institute. For the baseline assessment; PSG recording was performed over 2 nights. The first night served as an adaption night (no register); and 48hr later; the volunteers returned for another night of PSG recording. Each night; the volunteers arrived at the sleep laboratory at 21:00; and the exam began and finished based on each volunteer's habitual sleep schedule. The EMBLA (EMBLA S7000; Embla Systems Inc.; CO; USA) digital system was used. The measured variables included total sleep time (TST); sleep efficiencythe ratio between total sleep time and total recorded time multiplied by $100-(\mathrm{SE})$; sleep onset latency (SOL); wake after sleep onset (WASO); arousal index; sleep stages (I; II; III; and IV of NREM sleep and REM sleep); REM latency; and arterial oxygen saturation ( $\mathrm{SaO} 2)$. The apnea hypopnea index (AHI) and periodic leg movements (PLM) were also scored. A researcher; who was blinded to the study design; performed the staging and analyzed the polysomnography events using international criteria. ${ }^{13-15}$

Questionnaires: Approximately two days before the PSG assessment; the subjects arrived in the laboratory at 9 am to complete the Pittsburgh Sleep Quality Index (PSQI); ${ }^{16}$ Epworth Sleepiness Scale (ESS); ${ }^{17}$ Medical Outcomes Study SF-36-Quality of life; $;^{18}$ and Profile of Mood States (POMS). ${ }^{19}$

Pittsburgh Sleep Quality Index (PSQI): Sleep quality was determined using the PSQI. This questionnaire has seven items and a total possible score between 0 and 21. A PSQI score $\geq 5$ is considered to be an indicator of poor sleep quality. ${ }^{16}$

Epworth Sleepiness Scale (ESS): Daytime sleepiness was measured using the ESS. Respondents indicate on a four-point Likert scale $(0=$ never; $3=$ high chance $)$ the likelihood that they will "doze off or fall asleep" in eight different conditions. This scale has a total possible score from 0 to 24 . ESS scores $>10$ have been proposed to indicate excessive daytime sleepiness. ${ }^{17}$

Medical outcomes study SF-36-Quality of life: The SF-36 is a multidimensional questionnaire that covers eight components: physical functioning; role limitations caused by physical health problems; role limitations caused by emotional health problems; social functioning; vitality; general health perception; body pain; and mental health. All scores ranged from 0 to 100; with a higher score indicating better quality of life..$^{18}$ Profile of Mood States (POMS): The POMS questionnaire is an instrument for evaluating the profile of mood. It has 65 items and 6 domains: tension-anxiety; depression; anger-hostility; vigor-activity; fatigue; and confusion-bewilderment. The total mood disturbance score is derived by subtracting the vigoractivity score from the sum of the scores from the other subscales. ${ }^{19}$

Exercise testing: The participants were instructed to arrive at the laboratory in a rested and fully hydrated state; to not consume caffeine in the previous $4 \mathrm{~h}$; and to avoid strenuous exercise for the $48 \mathrm{~h}$ preceding a test session. To minimize the effects of diurnal biological variation; all of the tests were performed at the same time of day $(10: 00 \pm 2 \mathrm{~h})$. All participants underwent cardiopulmonary exercise testing (CPET) and a strength performance test; the one maximum repetition test $(1-\mathrm{RM}) .{ }^{20}$

The CPET was performed on a treadmill according to a modified
Bruce protocol (two stages of $2.7 \mathrm{~km} / \mathrm{h}$ with $0 \%$ and $5 \%$ grades; followed by the standard Bruce protocol). Respiratory gas samples were analyzed breath-to-breath using a portable metabolic system (K4b2;Cosmed SRL;Pavona;Rome;Italy). Before each test; the gas analyzers were calibrated according to the manufacturer's recommendations. Heart rate (HR) was recorded using a HR monitor (Polar Electronics; FS1; Kempele; Oulu; Finland). Peak oxygen uptake and HR max were defined as the mean oxygen uptake $\left(\mathrm{O}_{2}\right)$ and HR values; respectively; during the last 20 seconds of exercise. The maximal strength was tested using the 1-RM test (20) in the following strength devices: chest press and leg press (Technogym; Gambettola; Italy).

\section{Statistical analysis}

The program STATISTICA ${ }^{\circledR}$ (Statsoft; Inc; version 7.0) was used for data analysis. All variables had normal distributions $(\mathrm{p}>0.05)$ according to the normal probabilistic graph and KolmogorovSmirnov's test. We used Student's t test to compare the experimental and control groups and Pearson's correlation to verify the association between the AHI and the data from mood; quality of life and physical parameters. The data are expressed as the mean $\pm \mathrm{SD}$. The significance levels were set at $p \leq 0.05$.

\section{Results}

One hundred and twenty-four elderly men contacted the researchers with interest in participating in the study. Of this total; 84 were excluded for not meeting the inclusion criteria. Forty volunteers were included in the study protocol. They were allocated to either the OSA $(n=21)$ or the control group $(n=19)$.

The anthropometric and descriptive data for these volunteers are displayed in Table 1. There was no significant difference between the OSA and control groups for any of these measures. The CPET and 1-RM of the participants are shown in Table 2. No significant differences were observed in these variables. The polysomnographic parameters are presented in Table 3. There were significant differences in the AHI $(25.9 \pm 12.7$ vs. $7.9 \pm 4.7$ events/h); minimal oxygen saturation $(83.9 \pm$ .5 vs. $88.9 \pm 2.3 \%$ ); mean oxygen saturation ( $93.6 \pm 1.4$ vs. $94.5 \pm 1.2 \%$ ) and arousal index $(22.2 \pm 9.9$ vs. $13 ; 1 \pm 7 ; 0$ events/h) between the OSA and control groups $(\mathrm{p}<0 ; 05)$. Table 4 shows the data from the Quality of sleep (PSQI); Mood (POMS); Daytime sleepiness (Epworth) and Quality of life (SF-36) questionnaires. We observed similar behaviours between the groups in these parameters; except in the general health perception variable from the SF-36 questionnaire; in which the control group had a higher score $(87.5 \pm 12.8$ vs. $74.5 \pm 16.6(p<0.05)$. Additionally for the general health perception; there was a significant negative correlation; indicating an association between a large AHI and a low general health perception score $(r=-0.39 ; p<0 ; 05)$.

Table 1 Baseline characteristics of the lean elderly men with obstructive sleep apnea (OSA) and control group

\begin{tabular}{lll}
\hline & Control group $(\mathbf{n = 1 9})$ & $\begin{array}{l}\text { OSA group } \\
(\mathbf{n}=\mathbf{2 1})\end{array}$ \\
\hline Age (years) & $68.8 \pm 3.5$ & $69.8 \pm 3.5$ \\
Height $(\mathrm{cm})$ & $171.0 \pm 6.5$ & $167.3 \pm 6.5$ \\
Body mass $(\mathrm{kg})$ & $68,5 \pm 8.7$ & $66.3 \pm 7.6$ \\
BMI (kg.m-2) & $23.4 \pm 2.7$ & $23.6 \pm 1.8$ \\
MMSE (score) & $28.5 \pm 1.4$ & $27.9 \pm 0.8$ \\
\hline
\end{tabular}

BMI, body mass index; MMSE, mini mental state examination.

The results are presented as the mean \pm SD. $\mathrm{p}>0.05$. 
Table 2 Exercise testing data of lean elderly men with obstructive sleep apnea (OSA) and control group

\begin{tabular}{|c|c|c|}
\hline & Control group $(n=19)$ & OSA group $(n=21)$ \\
\hline \multicolumn{3}{|l|}{ CPET } \\
\hline$\dot{\mathrm{V}} O_{2 \text { peak }}\left(L \cdot \mathrm{min}^{-1}\right)$ & $2.1 \pm 0.4$ & $2.1 \pm 0.4$ \\
\hline $\begin{array}{l}\dot{\mathrm{V}} \\
O_{2 \text { peak }}\left(m L \cdot \mathrm{kg}^{-1} \cdot \mathrm{min}^{-1}\right)\end{array}$ & $30.9 \pm 5.0$ & $31.7 \pm 5.4$ \\
\hline $\mathrm{HR}_{\max }(\mathrm{beat} / \mathrm{min})$ & $150.9 \pm 13.3$ & $152.5 \pm 19.1$ \\
\hline Duration (min) & $16.3 \pm 1.7$ & $16.0 \pm 3.0$ \\
\hline \multicolumn{3}{|l|}{ 1-RM Test } \\
\hline Chest press (lb) & $87.4 \pm 16.9$ & $93.8 \pm 16.9$ \\
\hline Leg press (lb) & $236.8 \pm 55.5$ & $243.8 \pm 65.9$ \\
\hline \multicolumn{3}{|c|}{$\begin{array}{l}\text { CPET, cardiopulmonary exercise testing; } \\
\dot{V} \mathrm{O} 2 \text {, maximum oxygen uptake }\end{array}$} \\
\hline \multicolumn{3}{|c|}{ HRmax, peak heart rate } \\
\hline 1-RM, one maximum repetit & ion. & \\
\hline
\end{tabular}

Table 3 Polysomnographic data of lean elderly men with obstructive sleep apnea (OSA) and control group

\begin{tabular}{lll}
\hline & $\begin{array}{l}\text { Control group } \\
(\mathbf{n = 1 9})\end{array}$ & OSA group (n=21) \\
\hline AHI(events/h) & $7.9 \pm 4.7$ & $25.9 \pm 12.7^{*}$ \\
Total sleep time(min) & $317.6 \pm 54.2$ & $337.1 \pm 51.0$ \\
Stage 1(\%) & $6.7 \pm 3.9$ & $8.3 \pm 6.8$ \\
Stage 2(\%) & $58.3 \pm 7.8$ & $58.5 \pm 8.7$ \\
Stage 3 and 4(\%) & $14.4 \pm 5.1$ & $15.3 \pm 8.0$ \\
REM(\%) & $20.6 \pm 7.6$ & $17.8 \pm 6.9$ \\
Arousal index (events/h) & $13.2 \pm 7.0$ & $22.3 \pm 9.9 *$ \\
Sleep efficiency (\%) & $73.6 \pm 12.1$ & $77.4 \pm 9.7$ \\
SOL (min) & $16.9 \pm 14.7$ & $14.9 \pm 16.4$ \\
REM latency (min) & $87.1 \pm 40.6$ & $115.2 \pm 68.5$ \\
WASO (min) & $97.4 \pm 50.0$ & $82.7 \pm 38.3$ \\
PLM (events/h) & $1.5 \pm 3.6$ & $1.0 \pm 2.9$ \\
Lowest SaO $2 \%$ ) & $88.9 \pm 2.3$ & $83.9 \pm 5.3 *$ \\
Mean SaO $(\%)$ & $94.5 \pm 1.2$ & $93.6 \pm 1.4$ \\
\hline
\end{tabular}

AHI, apnea/hypopnea index; REM, rapid eye movement sleep; PLM, periodic leg movements; WASO, wake after sleep onset; $\mathrm{SaO} 2$, arterial oxygen saturation; SOL, sleep onset latency. The data are presented as the mean \pm SD. $* \mathrm{p}<0.05$.
Table 4 Subjective sleep quality, Sleepiness, Quality of life and Mood of lean elderly men with obstructive sleep apnea (OSA) and control group

\begin{tabular}{lll}
\hline & Control group $(\mathbf{n}=\mathbf{1 9})$ & OSA group $(\mathbf{n}=\mathbf{2 1})$ \\
\hline PSQI (score) & $6.2 \pm 3.3$ & $5.4 \pm 2.3$ \\
ESS (score) & $5.8 \pm 2.8$ & $8.2 \pm 4.7$ \\
SF-36 (Score) & & \\
Physical functioning & $92.1 \pm 7.3$ & $92.1 \pm 8.7$ \\
Role-physical & $93.1 \pm 11.5$ & $96.4 \pm 9.0$ \\
Body pain & $86.4 \pm 19.0$ & $85.7 \pm 17.0$ \\
General health & $87.5 \pm 12.8$ & $74.5 \pm 16.6^{*}$ \\
perception & $83.95 \pm 12.2$ & $80.0 \pm 11.5$ \\
Vitality & $96.0 \pm 10.2$ & $95.2 \pm 8.4$ \\
Social functioning & $91.2 \pm 24.4$ & $96.9 \pm 9.9$ \\
Role-emotional & $86.1 \pm 15.5$ & $80.8 \pm 10.9$ \\
Mental healthy & & \\
POMS (Score) & $3.7 \pm 4.5$ & $4.5 \pm 3.9$ \\
Tension-anxiety & $3.9 \pm 3.4$ & $5.5 \pm 4.8$ \\
Depression & $4.4 \pm 4.6$ & $6.4 \pm 6.0$ \\
Anger-hostility & $21.0 \pm 6.1$ & $20.5 \pm 4.3$ \\
Vigor-Activity & $2.0 \pm 2.1$ & $4.1 \pm 4.2$ \\
Fatigue & $0.1 \pm 3.1$ & $0.7 \pm 3.6$ \\
Confusion- & & \\
bewilderment & & \\
PSQ & &
\end{tabular}

PSQI, pittsburgh sleep quality index; ESS, epworth sleepiness scale; SF-36 short-form medical outcomes study; POMS, profile of mood states. The data are presented as the mean \pm SD. ${ }^{*} \mathrm{p}<0.05$.

\section{Discussion}

The aim of this study was to compare sleep quality; daytime sleepiness; exercise capacity; mood and quality of life of lean elderly men with Obstructive Sleep Apnea (OSA) vs non-sleep apnea. As expected; we observed significant difference in the apnea/hypopnea index; minimal oxygen saturation; mean oxygen saturation and arousal index between groups. However; an increase in stages 1 and 2 and reduction in stages 3 and 4 in both groups were observed comparing with the standard sleep criteria for adults. These alterations could be justified by aging; which is associated with superficial sleep; increments in stages 1 and $2^{21,22}$ and a reduction in slow wave sleep caused by decreased delta amplitude. ${ }^{23,24}$ The mean percentage of REM sleep decreased in the OSA subjects but not in the control group; which had a normal percentage (approximately 20;6\%) considering the standard criteria for adults $(20-25 \%) .{ }^{15}$ The OSA and control groups also had lower sleep efficiency considering standard criteria for adults $(>85 \%)$. According to Ohayon et al. ${ }^{22}$ sleep efficiency is the only parameter that remains reduced in the elderly. Other parameters such as sleep onset latency; REM latency; wake after sleep onset and total sleep time are normal or slightly modified in both groups; but this slight change is common in the elderly. ${ }^{21}$

As soon as polysomnography data; the PSQI suggest that sleep quality of OSA group was not impacted. Probably this finding has been result of strict inclusion and exclusion criteria. The participants included in OSA group; despite sleep apnea had no other impairments on general health; as obesity; functional impairments or uncontrolled illness.

We also observed similar performance during the exercise testing in the OSA and control group. In addition; the observed $\mathrm{O}_{2}$ peak value were into normality considering age and gender. ${ }^{25}$ Consistent with our results; Rizzi et al. ${ }^{7}$ reported no difference when comparing the 
exercise capacity of lean middle-age subjects with and without OSA. In contrast; other studies ${ }^{26,27}$ have reported a diminished $\mathrm{O} 2$ peak in obese patients with OSA. Thus; it appears that obesity is an important factor in and could be responsible for these impairments.

At strength performance; we observed no differences between OSA and control group. Although we have not found other studies evaluating strength performance in OSA patients; the results of the present study show that lean elderly men with OSA do not have major impairments in general physical capacity.

Although many studies have presented associations between OSA and impairments in daytime functions in middle-age patients; ${ }^{10}$ the elderly may present a different behavior. Some studies have demonstrated low or no association between OSA and daytime impairments such as excessive daytime sleepiness or mood disorders. ${ }^{28,29}$ Consistent with these studies; we observed no difference in the subjective mood and daytime sleepiness. Depression is the major mood disorder associated with OSA..$^{30}$ According to El-Ad et al. ${ }^{31}$ the severity of depression is correlated to daytime sleepiness more than to hypoxemia. The daytime sleepiness evaluated in the present study was within the normality criteria $(<10 \mathrm{events} / \mathrm{h})$; supporting our results.

The quality of life of OSA and control group was similar; except in the general health perception. However; the mean values of all SF-36 domains are higher than the reference values reported in other studies; adjusted for age and gender. ${ }^{32,33}$ In the Sleep Heart Health Study data $;{ }^{9}$ a linear association of OSA severity with decrements in the eight SF-36 scales was observed. However; the authors observed this association only when the OSA was defined as an AHI $>30$. According to a recent study $;^{34}$ the quality of life may be poor in patients with sleep apnea for multifactorial reasons. The authors examined OSA patients and observed a negative correlation among most domains of quality of life with sleepiness; body mass index; hypertension and glucose metabolism disorders. It is possible that body mass index and daytime sleepiness explain the observed negative association between OSA and quality of life. These parameters were normal in our study (lean subjects with no daytime sleepiness).

There was a considerable limitation in the present study. The subjective scales used to evaluate sleep quality; daytime sleepiness; quality of life and mood have not been developed specifically for the elderly. However; some large studies using subjects with or without OSA have used these scales. ${ }^{32,35-37}$ Another possible limitation could be our strict inclusion and exclusion criteria. All participants were in good physical and mental health. This condition cannot be common in many OSA patients; considering that OSA is frequently associated with other illness.

\section{Conclusion}

In conclusion; our results suggest that OSA does not impact significantly the sleep quality; daytime sleepiness; physical capacity; mood and quality of life of lean elderly men. We could hypothesize that daytime impairments in OSA patients are mediated by body mass index.

\section{Acknowledgements}

None.

\section{Conflicts of interest}

The author declared that there are no conflicts of interest.

\section{References}

1. Tufik S, Santos-Silva R, Taddei JA, et al. Obstructive sleep apnea syndrome in the Sao Paulo epidemiologic sleep study. Sleep Med. 2010;11(5):441446 .

2. Young T, Peppard PE, Gottlieb DJ. Epidemiology of obstructive sleep apnea: a population health perspective. Am J Respir Crit Care Med. 2002;165(9):1217-1239.

3. The international classification of sleep disorders: diagnostic and coding manual. Diagnostic classification steering committee, Westchester, llinois, USA: American academy of sleep medicine; 2005.

4. Newman AB, Foster G, Givelber R, et al. Progression and regression of sleep-disordered breathing with changes in weight: the sleep heart health study. Arch Intern Med. 2005;165(20):2408-2413.

5. Davies CT, Godfrey S, Light $\mathrm{M}$, et al. Cardiopulmonary responses to exercise in obese girls and young women. J Appl Physiol. 1975;38(3):373376.

6. Serés L, López-Ayerbe J, Coll R, et al. Cardiopulmonary function and exercise capacity in patients with morbid obesity. Rev Esp Cardiol. 2003;56(6):594-600

7. Rizzi CF, Cintra F, Risso T, et al. Exercise capacity and obstructive sleep apnea in lean subjects. Chest. 2010;137(1):109-114.

8. American Academy of Sleep Medicine. Obstructive Sleep Apnea. The Academy, Illinois, USA: AASM; 2008.

9. Baldwin CM, Griffith KA, Nieto FJ, et al. The association of sleepdisordered breathing and sleep symptoms with quality of life in the sleep heart health study. Sleep. 2001;24(1):96-105.

10. Young T, Palta M, Dempsey J, et al. The occurrence of sleep-disordered breathing among middle-aged adults. $N$ Engl J Med. 1993;328(17):1230 1235 .

11. Zortea K, Silva ML. Body mass index in adult and elderly individuals. Arq Bras Cardiol. 2011;96(3):255.

12. Folstein MF, Folstein SE, McHugh PR. Mini-mental state. A practical method for grading the cognitive state of patients for the clinician. $J$ Psychiatr Res.1975;12(3):189-198.

13. American sleep disorders association. EEG arousals: scoring rules and examples: a preliminary report from the Sleep Disorders Atlas task force of the American sleep disorders association. Sleep. 1992;15(2):173-184.

14. American sleep disorders association. Sleep-related breathing disorders in adults: recommendations for syndrome definition and measurement techniques in clinical research. The report of an American academy of sleep medicine task force. Sleep. 1999;22(5):667-689.

15. Rechtschaffen A, Kales A. A manual of standardized terminology, techniques and scoring system for sleep stages of human subjects. Washington, USA; 1968

16. Buysse DJ, Reynolds CF, Monk TH, et al. The pittsburgh sleep quality index: a new instrument for psychiatric practice and research. Psychiatry Res. 1989;28(2):193-213.

17. Johns MW. A new method for measuring daytime sleepiness: the Epworth sleepiness scale. Sleep. 1991;14(6):540-545. 
18. Da Mota Falcão D, Ciconelli RM, et al. Translation and cultural adaptation of quality of life questionnaires: an evaluation of methodology. $J$ Rheumatol. 2003;30(2):379-385.

19. McNair DM LM, Droppelman LF. Manual for the Profile of Mood States San Diego, California, USA: Education and industrial testing service; 1971.

20. Kraemer WJ, Ratames NA, Fry AC, et al. Strength testing: development and evaluation of methodology. In: Maud PJ, et al, editors. Physiological Assessment of Human Fitness. 2nd ed. Champaign, Illinois, USA: Human Kinetics; 2006. p. 119-150.

21. Bliwise DL. Principles and practice of sleep medicine. Philadelphia, USA Saunders; 2000

22. Ohayon MM, Carskadon MA, Guilleminault C, et al. Meta-analysis of quantitative sleep parameters from childhood to old age in healthy individuals: developing normative sleep values across the human lifespan. Sleep. 2004;27(7):1255-1273.

23. Bliwise DL, Bergmann BM. Individual differences in stages 3 and 4 sleep. Psychophysiology. 1987;24(1):35-40.

24. Feinberg I, Thode HC Jr, Chugani HT, et al. Gamma distribution model describes maturational curves for delta wave amplitude, cortical metabolic rate and synaptic density. $J$ Theor Biol. 1990;142(2):149-161.

25. Medicine ACoS. ACSM's Guidelines for Exercise Testing and Prescription Philadelphia, Pennsylvania, USA: Lippincott Williams \& Wilkins; 2009.

26. Lin CC, Hsieh WY, Chou CS, et al. Cardiopulmonary exercise testing in obstructive sleep apnea syndrome. Respir Physiol Neurobiol. 2006;150(1):27-34.

27. Vanhecke TE, Franklin BA, Zalesin KC, et al. Cardiorespiratory fitness and obstructive sleep apnea syndrome in morbidly obese patients. Chest. 2008;134(3):539-545.
28. Asghari A, Mohammadi F, Kamrava SK, et al. Severity of depression and anxiety in obstructive sleep apnea syndrome. Eur Arch Otorhinolaryngol. 2012;269(12):2549-2553.

29. Harris M, Glozier N, Ratnavadivel R, et al. Obstructive sleep apnea and depression. Sleep Med Rev. 2009;13(6):437-444.

30. Baran AS, Richert AC. Obstructive sleep apnea and depression. CNS Spectr. 2003;8(2):128-134.

31. El-Ad B, Lavie P. Effect of sleep apnea on cognition and mood. Int Rev Psychiatry. 2005;17(4):277-282.

32. López-García E, Banegas JR, Graciani Pérez-Regadera A, et al [Population-based reference values for the Spanish version of the SF-36 Health Survey in the elderly]. Med Clin (Barc). 2003;120(15):568-573.

33. Martinez-Garcia MA, Soler-Cataluna JJ, Roman-Sanchez P, et al Obstructive sleep apnea has little impact on quality of life in the elderly. Sleep Med. 2009;10(1):104-111.

34. Bulcun E, Ekici A, Ekici M. Quality of life and metabolic disorders in patients with obstructive sleep apnea. Clin Invest Med. 2012;35(2):E105113.

35. Hayes V, Morris J, Wolfe C, et al. The SF-36 health survey questionnaire: is it suitable for use with older adults? Age Ageing. 1995;24(2):120-125.

36. Seymour DG, Ball AE, Russell EM, et al. Problems in using health survey questionnaires in older patients with physical disabilities. The reliability and validity of the SF-36 and the effect of cognitive impairment. $J$ Eval Clin Pract. 2001;7(4):411-418.

37. Kales A, Wilson T, Kales JD, et al. Measurements of all-night sleep in norma elderly persons: effects of aging. J Am Geriatr Soc. 1967;15(5):405-414. 